\section{The term risk: etymology, legal definition and various traits}

\author{
Gaetano Liuzzo, ${ }^{1}$ Stefano Bentley, ${ }^{2}$ \\ Federica Giacometti, ${ }^{3}$ Elena Bonfante, ${ }^{3}$ \\ Andrea Serraino ${ }^{3}$
}

${ }^{1}$ Azienda Unità Sanitaria Locale di Modena, Distretto di Carpi (MO); ${ }^{2}$ Dipartimento di Salute Animale, Università di Parma; ${ }^{3}$ Dipartimento di Scienze Mediche Veterinarie, Alma Mater Studiorum - Università di Bologna, Ozzano dell'Emilia (BO), Italy

\section{Abstract}

The etymology of the term risk and its legal qualification and definitions are reported in this article; decription of the various traits of the term risk used in food safety management (acceptable risk, current risk, emerging risk, crude risk, unrestricted risk, perceived risk, real risk, residual risk, reduced risk, baseline risk, serious risk, major technological risk, etc.) are presented and discussed.

\section{English version}

\section{Risk (etymology)}

The word has probably two origins, and both are related to the concept of danger; risk might be traced back to the Italian word risco or either to the Spanish riesco, both deriving from the latin resecum (the one that cuts), a nautical expression used to define the cliffs which threatened the ships and any danger to sailors and naval trades. This etymology links the word to the sea insurance companies which used to be common in Genoa back in $14^{\text {th }}$ century. The other possible etymology is linked to the Romance word rixicare (argue, debate, to put up a fight) (Peretti-Watel, 2001).

\section{Risk (legal qualification)}

The German jurisprudence, and later the French, classifies the risk in three major groups in regard to precautionary issues: i) certain/unacceptable risks, whose cause-effect link between event and damage is scientifically proven, with the only doubt regarding when; to these risks the prevention principle is applied; ii) residuaVAcceptable risks, deriving from normal human activities, resting upon mere speculations and not supported by science-based evidence (hypothetical risks, which cannot be taken into account in decisional processes, not to determine stall-like effects, nor discriminatory conclusions); and iii) uncertain risks, not yet scientifically proven, but whose existence cannot be ruled out. This category is relevant for precautionrelated issues (Marini et al., 2004).

\section{Definition in the European}

Commission food safety legislation

According to Regulation (EC) No 178/2002 of the European Parliament and of the Council of 28 January 2002 risk means a function of the probability of an adverse health effect and the severity of that effect, consequential to a hazard. Most credited definition of risk defines it as a function of the likelihood of an adverse health effect and the severity of that effect, consequential to a hazard in a food (FAO, 1995; CAC, 2004; European Commission, 2002). With a slight difference, OIE defines it as the likelihood of the occurrence and the likely magnitude, given a certain time, of the biological and economic consequences of an adverse event to animal or human health in the import country, both in biological and economical terms (OIE, 2009). Ever since these first indications, it can be noticed how in food safety issues, Codex highlights the adverse health effects, while World Animal Health Organization, formerly Office International des Épizooties includes both biological and economical outcomes.

In both definitions, however, two main elements can be found: in food, the likelihood and severity on health effects, while in animal health, likelihood and importance of consequences (Cerf, 2008). It is a quantitative notion, based on two elements: the frequency of hazard occurrence and the magnitude of its outcomes. The risk level of a hazard is a function of these two components. It's generally easier to estimate the former, i.e. the incidence is the measured frequency, while the risk is the thereby derived possible frequency (Cerf et al., 1996). Thus, risk can be visualized with the expression $\mathrm{R}=\mathrm{MxF}$ (M for magnitude, or severity of outcomes, and $\mathrm{F}$ for frequency). When using an emotional-perceptive approach, such as for communication issues, the formula is redefined into $\mathrm{R}=\mathrm{HxO}$ ( $\mathrm{H}$ for hazard and 0 for Outrage (Sandman, 1987).

\section{Acceptable risk}

According to OIE (2009), it is a risk level judged by each OIE Member to be compatible with the protection of animal and public health within its territory (Toma et al., 2002).

\section{Current risk}

Risk at the beginning of the risk evaluation process. This risk may, in certain cases, be a crude risk and, in other cases, a partially reduced risk by prior implementation of certain management measures (AFSSA, 2008).

\section{Emerging risk}

A potential hazard linked to food and feed
Correspondence: Andrea Serraino, Dipartimento di Scienze Mediche Veterinarie, Alma Mater Studiorum, Università di Bologna, Ozzano dell'Emilia (B0), Italy.

Tel.+39.051.2097332 - Fax: +39.051.2097346.

E-mail: andrea.serraino@unibo.it

Key words: Risk, Legal definition, Food safety.

Received for publication: 4 February 2014. Accepted for publication: 5 February 2014.

This work is licensed under a Creative Commons Attribution 3.0 License (by-nc 3.0).

(C) Copyright G. Liuzzo et al., 2014

Licensee PAGEPress, Italy

Italian Journal of Food Safety 2014; 3:2269

doi:10.4081/ijfs.2014.2269

that might become a risk for public health; though mentioned in EC Regulation 178/2002, no technical or legal definition of emerging risk is provided. As for the technical issue, the gap was filled by the EFSA, which defined it (EFSA, 2007) and lays tools and principles for its identification (EFSA, 2010, 2012). Emerging risk for human, animal and plant health can be the result of: significant exposure to a newly identified hazard, which was previously non existing or not known; re-emerging hazards (i.e. already known), for which an unexpected or increased exposure may occur; increased significant susceptibility to a known hazard in humans, animals and plants. In this context, the term new can be interpreted as new scientific evidence, or not regulated in the EU, or not yet addressed by EFSA. The term identified can be interpreted in different ways depending on the type and amount of supporting evidence considered necessary, with the general understanding that false positive emerging risks should be limited. The emerging risks' identification is based on data (inevitably and typically insufficient and incomplete), which nonetheless should be as much as possible solid, although initial and not exclusively on theoretical speculations. The term significant, in this context, is used in the sense of meaningful or relevant and not statistically significant. The evaluation of significant exposure is a cornerstone in the emerging risks identification, and its definition is necessary. The detection of a high number of false positive emerging risks by EFSA, and the thereby arising problems, suggested the definition of emerging issue: an emerging issue is an issue with similar characteristics of an emerging risk, but for which the information collected is preliminary and too limited to be able to assess whether it is (or it could develop) into an emerging risk. The speed of development of an emerging risk affects the approach (slow or fast), of its identification. 


\section{Crude risk}

Estimated risk when no management measure has been taken (AFSSA, 2008).

\section{Unrestricted risk (also called inher- ent risk)}

The level of risk that would be present if no specific actions were taken to control it (WHO/FAO, 2009), or the risk which would exist in the absence of current controls (Shaw, 2003). The concept is widely used in import risk analysis. It may have a role in comparing the efficacy of existing risk management approaches with entirely new ones.

\section{Perceived risk}

Risk resulting from an individual, subjective assessment, based on a mental representation (Ruminati and Salvadori, 1999).

\section{Real risk}

Objective, unbiassed risk, result of an actuarial-probabilistic assessment (Ruminati and Salvadori, 1999).

\section{Residual risk}

Refers to the current level of risk, assuming reasonable compliance with current regulations and other controls (Shaw, 2003).

\section{Reduced risk}

Estimated risk taking account of new management measures (AFSSA, 2008).

\section{Baseline risk}

The current, standard or reference risk status, i.e. the point against which the benefits and costs of various intervention strategies can be compared. A common and practical starting point for a risk assessment is to estimate the existing level of risk, i.e. the level of food safety risk, thus obtaining the baseline risk, against which intervention strategies can be valued. This baseline risk may, for example be useful in determining an Appropriate Level of Protection (ALOP) (WHO/FAO, 2009).

\section{Serious risk}

Direct or indirect risk to human health and/or is perceived or publicised as such or can be perceived and/or publicised as such, which is spread or could be spread by a large part of the food chain with a high potential likelihood of spreading to several Member States and/or non-Community countries (European Commission, 2004).

\section{Major technological risk}

Low likelihood of occurrence, but with catastrophic consequences, such as the loss of human lives, material and financial losses or other damages to the Community. It is a technology-linked risk (Lagadec, 1981).

\section{Italian version}

\section{Rischio (etimologia)}

Sono due le possibili origini etimologiche della parola rischio ed entrambe evocano l'idea di pericolo. Rischio deriverebbe dall'italiano risco o dallo spagnolo riesco, entrambe derivanti dal latino resecum (lett. ciò che taglia), per designare dapprima lo scoglio che minaccia i navigatori e poi in generale il pericolo corso dai mercanti in mare. Questa etimologia associa il rischio alle assicurazioni marittime che apparvero in Italia, soprattutto a Genova, nel XIV secolo. L'altra origine etimologica possibile è dal termine romanzo rixicare cioè litigare, di ciò che ha provocato una rissa (Peretti-Watel, 2001).

\section{Rischio (qualificazione giuridica)}

La dottrina tedesca propone una classificazione del rischio cui si rivolge la precauzione, secondo questa dottrina, ripresa anche da quella francese, è possibile distinguere: i) i rischi certi 0 inaccettabili, rispetto ai quali il nesso di causalità tra l'evento e il danno è provato scientificamente, anche se restano dubbi sui tempi in cui si verificherà l'evento in questione (a tali rischi si applica il principio di prevenzione); ii) i rischi residuali 0 accettabili, derivanti dal normale svolgimento delle attività umane, che riposano su considerazioni puramente speculative e non appaiono suffragati da fondamenti scientifici (si tratta di rischi ipotetici, che non possono essere assunti in considerazione nell'ambito dei processi decisionali per non determinare effetti paralizzanti o discriminatori); iii) i rischi incerti, non ancora dimostrati dalla scienza, ma di cui non è irragionevole supporre l'esistenza. questa la categoria dei rischi che viene in rilievo ai fini della precauzione (Marini, 2004).

\section{Definizione nella legislazione Comunitaria sulla sicurezza alimentare}

Secondo il Regolamento (CE) 178/2002 del Parlamento europeo e del Consiglio, del 28 gennaio 2002, che stabilisce i principi e i requisiti generali della legislazione alimentare, istituisce l'Autorità europea per la sicurezza alimentare e fissa procedure nel campo della sicurezza alimentare, il termine rischio definisce la funzione della probabilità e della gravità di un effetto nocivo per la salute, conseguente alla presenza di un pericolo.

La maggior parte delle definizioni accreditate definiscono il rischio come funzione della probabilità e della gravità di un effetto nocivo per la salute, conseguente alla presenza di un pericolo in un alimento (FAO, 1995; CAC, 2004; European Commission, 2002). Con qualche differenza l'OIE definisce invece il rischio come la probabilità di sopravvenienza e l'am- piezza probabile, nel corso di un determinato periodo, delle conseguenza di un avvenimento pregiudizievole alla salute animale e umana nel paese importatore in termini biologici ed economici (OIE, 2009). Da queste prime indicazioni si nota come nel contesto specifico della sicurezza degli alimenti viene considerata, in base alla definizione del Codex, solo la conseguenza riferibile agli effetti avversi alla salute. Dalla definizione dell'Office International des Épizooties, oggi Organizzazione Mondiale della Sanità Animale, risultano essere invece di due tipi le conseguenze comprese nella definizione di rischio: biologiche ed economiche. In entrambe i contesti, sempre due gli elementi essenziali: nel contesto degli alimenti, la probabilità e la gravità degli effetti sulla salute, mentre nel contesto della salute animale, la probabilità e la grandezza (importanza) delle conseguenze (Cerf, 2008). Si tratta di una nozione quantitativa in cui sono individuabili due componenti: la frequenza di comparsa del pericolo e l'importanza delle conseguenze del pericolo. Il livello di rischio di un pericolo è funzione della probabilità di queste due componenti. Spesso è più facile stimare la componente legata alla frequenza. In effetti l'incidenza è la frequenza misurata, il rischio è la frequenza possibile 0 probabile, dedotta dalla frequenza misurata (Cerf et al., 1996). In base a questa definizione di tipo meccanicistico, prodotto della ricerca scientifica possiamo descrivere il rischio con l'espressione simbolica: $\mathrm{R}=\mathrm{MxF}$ dove $\mathrm{M}$ sta per magnitudine o magnitudo, ossia gravità delle conseguenze ed $\mathrm{F}$ per frequenza. Il termine rischio viene riconcettualizzato secondo un approccio percettivo-emozionale ai fini della sua comunicazione. L'espressione simbolica che lo descrive diventa quindi: $\mathrm{R}=\mathrm{Px} 0$ dove $P$ sta per pericolo ed 0 per offesa (Sandman, 1987).

\section{Rischio accettabile}

Il livello di rischio giudicato compatibile con la salute, tenuto conto di un insieme di dati epidemiologici, sociali ed economici (Toma et $a l ., 2002)$. Secondo l'OIE è il livello di rischio che un membro dell'OIE giudica compatibile con la protezione della salute pubblica e della sanità animale sul suo territorio (OIE, 2009).

\section{Rischio attuale}

Il rischio nel momento di inizio di un processo di valutazione, può essere in certi casi grezzo, ed in altri casi, parzialmente ridotto per la messa in opera di determinate azioni di gestione (AFFSA, 2008).

\section{Rischio emergente}

Un pericolo potenziale legato alle derrate alimentari 0 ai mangimi che può nel futuro (avvenire prossimo) diventare un rischio per la salute pubblica. Nonostante sia citato nel citato Regolamento (CE) 178/2002, manca una 
definizione sia tecnica che legale del termine. Questa lacuna viene colmata, per la parte tecnica, dall'EFSA che ne dà dapprima una definizione (EFSA, 2007) e successivamente ne definisce i principi e gli strumenti per la sua identificazione (EFSA, 2010, 2012). Un rischio emergente per la salute umana, animale e/o delle piante è il rischio causato dal realizzarsi di tre possibili situazioni. Il rischio emergente può conseguire: alla significativa esposizione ad un pericolo identificato recentemente e che prima o non esisteva o non era conosciuto, un pericolo nuovo; al riemergere di un pericolo conosciuto per il quale si realizza una inaspettata 0 maggiore esposizione; alla maggiore sensibilità da parte di persone, animali e/o piante, nei confronti di un pericolo conosciuto. In questo contesto, il termine nuovo può essere interpretato come una nuova evidenza scientifica, una evidenza non ancora regolata dall'Unione Europea od ancora, una evidenza non sottoposta al vaglio dell'EFSA. Il termine identificato si può interpretare in vari modi a seconda del tipo e della quantità di evidenze scientifiche di supporto considerate necessarie e con la generale considerazione che, nel limite del possibile, sono da limitare i rischi emergenti falsi positivi. L'identificazione dei rischi emergenti (ERI) si basa su dati, inevitabilmente e tipicamente insufficienti e incompleti, che devono comunque essere il più possibile solidi anche se iniziali, non dovranno comunque basarsi su speculazioni teoriche. Il termine significativo deve essere interpretato nel senso di importante, rilevante ma non nel senso di statisticamente significativo. La valutazione dell'esposizione (significativa) è un elemento di fondante importanza e la sua definizione è indispensabile ai fini dell'identificazione di un rischio emergente. Il rilievo da parte dell'EFSA di un alto numero di cd. Rischi emergenti falsi positivi ha suggerito la definizione del concetto di problema emergente. Si parla di problema emergente riferendosi ad un problema con caratteristiche simili a quelle di un Rischio Emergente ma per il quale le informazioni raccolte sono preliminari e limitate tanto da non rendere possibile la valutazione finalizzata a stabilire se si tratta 0 potrebbe trattarsi di un Rischio emergente. La velocità di sviluppo di un rischio emergente comporta come conseguenza un diverso approccio, più 0 meno rapido, della sua identificazione.

\section{Rischio grezzo}

Il rischio stimato nel momento in cui non è stata presa e messa in atto nessuna misura di gestione (AFSSA, 2008).

\section{Rischio non limitato (o rischio iner- ente)}

Il livello di rischio che sarebbe presente se non vi fosse alcun tipo di misura specifica per ridurlo (WHO/FAO, 2009), che potrebbe esiste- re in assenza di controlli correnti (Shaw, 2003). Il concetto è largamente utilizzato nelle Analisi del Rischio all'importazione. Può risultare utile per confrontare l'efficacia di un approccio esistente di gestione del rischio con un approccio radicalmente diverso e/o nuovo.

\section{Rischio percepito}

Rischio prodotto della valutazione degli individui, soggettivo, basato sulla rappresentazione mentale (Ruminati e Salvadori, 1999).

\section{Rischio reale}

Rischio oggettivo, obiettivo e valutato, prodotto dell'analisi attuariali-probabilistiche (Ruminati e Salvadori, 1999).

\section{Rischio residuale}

Si riferisce al livello di rischio corrente, assumendo una ragionevole conformità alle regole correnti e ad altri controlli (Shaw, 2003).

\section{Rischio ridotto}

Il rischio stimato tenendo conto della applicazione di nuove misure di gestione (AFSSA, 2008).

\section{Rischio di riferimento}

Il rischio che corrisponde alla situazione attuale 0 alla norma, cioè a dire il punto in rapporto al quale si possono comparare costi e vantaggi delle diverse strategie d'intervento. In una Valutazione del Rischio solitamente si inizia valutando il livello di un rischio esistente, cioè di un rischio che incide sulla sicurezza sanitaria degli alimenti, così facendo si ottiene il rischio di riferimento in rapporto al quale possono essere valutate le strategie d'intervento. In questo modo il rischio di riferimento può essere utile per determinare il Livello Appropriato di Protezione (LAP) (WHO/FAO, 2009).

\section{Rischio serio}

Quel rischio diretto o indiretto per la salute umana e/o percepito o divulgato come tale che si diffonde 0 potrebbe diffondersi attraverso una parte considerevole della catena alimentare e la cui potenziale ampiezza di coinvolgimento di più Stati membri e/o paesi terzi è notevole (European Commission, 2004).

\section{Rischio tecnologico maggiore}

Probabilità e/o eventualità che l'avvenimento che si realizza è scarsa ma le conseguenze sono catastrofiche e suppongono la perdita di vite umane, perdite materiali e finanziarie od altro per la collettività. È un rischio legato alle tecnologie (Lagadec, 1981).

\section{References}

AFSSA, 2008. [Une méthode qualitative d'esti- mation du risque en santé animale. Rapport du comité d'experts spécialisé «Santé Animale» de l'AFSSA]. [Book in French]. Agence française de sécurité sanitaire des aliments ed., Maison Alfort, France.

CAC, 2004. [Textes de base concernant l'hygiene alimentaire]. [Book in French]. Commission du Codex Alimentarius ed., Rome, Italy. Available from: http://www. fao.org/ag/agn/CDfruits_fr/others/docs/CA C-RCP1-1969.PDF

Cerf 0, 2008. Current definitions of risk for food safety and animal health allow risk assessment to provide substantially different outcomes. Risk Anal 28:811-3.

Cerf O, Sanaa M, Dufour B, Toma B, 1996. [Nomenclature proposée pour l'analyse de risque]. [Article in French]. Rev Epid San Anim 30:35-43.

EFSA, 2007. Definition and description of "Emerging Risks" within the EFSA's mandate (adopted by the Scientific Committee on 10 July 2007). European Food Safety Authority ed., Parma, Italy.

EFSA, 2010. Development and implementation of a system for the early identification of emerging risks in food and feed. EFSA Journal 8:1888.

EFSA, 2012. Towards a methodological framework for emerging risk identification. European Food Safety Authority ed., Parma, Italy. Available from: http://www. efsa.europa.eu/it/search/doc/243e.pdf

European Commission, 2002. Regulation of the European Parliament and of the Council of 28 January 2002 laying down the general principles and requirements of food law, establishing the European Food Safety Authority and laying down procedures in matters of food safety, 178/2002/CE. In: Official Journal, L 31/1, 01/02/2002.

European Commission, 2004. Commission Decision of 29 April 2004 concerning the adoption of a general plan for food/feed crisis management. 478/2004/CE. In: Official Journal, L 212/60, 12/06/2004.

FA0, 1995. [Application de l'analyse des risques dans le domaine des normes alimentaires]. [Report in French]. Food and Agriculture Organisation ed., Geneva, Switzerland.

Lagadec P, 1981. [La civilisation du risque. Catastrophes technologiques et responsabilité sociale]. [Book in French]. Le Seuil ed., Paris, France.

Marini L, 2004. [Il principio di precauzione nel diritto internazionale e comunitario]. [Book in Italian]. CEDAM, Padova, Italy.

OIE, 2009. [Code sanitaire pour les animaux terrestres]. [Book in French]. Organisation Mondiale de la Santé Animale ed., Paris, France.

Peretti-Watel P, 2001. [La société du risque]. 
[Book in French]. La Découverte ed., Paris, France.

Rumiati R, Savadori L, 1999. [Percezione del rischio e rischio tecnologico-professionale]. [Book in Italian]. Risorsa Uomo 6:722.

Sandman PM, 1987. Risk communication: facing public outrage. Available from: http://classwebs.spea.indiana.edu/dhenshel/sandman.pdf

Shaw I, 2003. Ranking food safety risks: a discussion document. Institute of Environmental Science \& Research Ltd. ed., Christchiurch, New Zealand.

Toma B, Dufour B, Sanaa M, 2002. [Generalités sur l'analyse du risque]. [Article in
French]. Rev Epid San Anim 41:5-16.

WHO/FAO, 2009. [Caractérisation des risques liés aux dangers microbiologiques d'origine alimentaire]. [Book in French]. World Health Organisation ed., Rome, Italy. Available from: ftp://ftp.fao.org/ ag/agn/jemra/17f.pdf 